Recherches en didactique des langues et des cultures

Les cahiers de l'Acedle

17-2 | 2020

Recherches collaboratives en didactique des langues

\title{
Pourquoi, quoi, comment... et après ? Regards de chercheure et d'enseignant sur un projet collaboratif en éducation autochtone
}

Eva Lemaire, René Beauparlant et Cécile Howse

\section{(2) OpenEdition \\ 12 Journals}

Édition électronique

URL : https://journals.openedition.org/rdlc/7537

DOI : $10.4000 /$ rdlc. 7537

ISSN : 1958-5772

Éditeur

ACEDLE

Référence électronique

Eva Lemaire, René Beauparlant et Cécile Howse, «Pourquoi, quoi, comment... et après ? Regards de chercheure et d'enseignant sur un projet collaboratif en éducation autochtone », Recherches en didactique des langues et des cultures [En ligne], 17-2 | 2020, mis en ligne le 27 avril 2020, consulté le 17 octobre 2022. URL : http://journals.openedition.org/rdlc/7537 ; DOI : https://doi.org/10.4000/rdlc. 7537

Ce document a été généré automatiquement le 17 octobre 2022

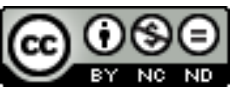

Creative Commons - Attribution - Pas d'Utilisation Commerciale - Pas de Modification 4.0 International - CC BY-NC-ND 4.0

https://creativecommons.org/licenses/by-nc-nd/4.0/ 


\title{
Pourquoi, quoi, comment... et après ? Regards de chercheure et d'enseignant sur un projet collaboratif en éducation autochtone
}

\author{
Eva Lemaire, René Beauparlant et Cécile Howse
}

\section{Introduction}

1 Les recherches participatives, dans la lignée des travaux d'Anadón (2007) et de Bourrassa et al. (2012), sont de plus en plus souvent perçues en sciences de l'éducation comme des recherches engagées, devant apporter des solutions collectives à des problèmes identifiés en commun. Le courant émergeant de la recherche anglo-saxonne dite «community-based " nous amène à repenser plus en profondeur encore ce que peuvent être l'engagement et la posture du chercheur, tant il est vrai que les recherches communautaires nord-américaines allient désormais recherche appliquée et activisme social (Koster et al., 2012 ; Strand et al., 2003).

2 Le projet de recherche dont il est ici question se situe à la croisée de deux influences distinctes, mais complémentaires. S'il constitue une recherche en éducation, impliquant le terrain scolaire, il repose également sur une collaboration avec la communauté autochtone, dans une optique de réconciliation et de revitalisation d'une langue en voie de disparition, la langue michif.

3 Dans cet article, nous commencerons par expliquer plus en détail le paysage sociétal et éducatif dans lequel se situe la recherche. Nous décrirons ensuite le projet, avec sa phase initiale impliquant la communauté métisse ${ }^{3}$ puis sa phase de recherche-actionformation auprès des enseignants (Charlier, 2005 ; Paillet, 1994). Après nous être présentés, nous nous concentrerons sur la collaboration spécifique que nous avons 
établie, E. Lemaire, comme chercheure ${ }^{4}$ d'une part, et $\mathrm{R}$. Beauparlant comme enseignant d'autre part. Le texte abordera ensuite la posture singulière de la chercheure, qui joue un rôle de pont entre la communauté scolaire et la communauté autochtone, à travers notamment la voix de $C$. Howse, aînée ${ }^{5}$ et locutrice de michif. On interrogera enfin les responsabilités et questionnements éthiques avec lesquelles composer dans ce contexte.

\section{Le contexte de la réconciliation entre peuples autochtones et non autochtones}

\section{Quelques repères}

4 Afin d'esquisser le portrait d'une situation sociétale extrêmement complexe, nous commencerons par les excuses publiques du premier ministre canadien, Stephen Harper, formulées en 2008. Le ministre reconnaît alors "un triste chapitre » de l'histoire du Canada, s'excusant pour le système des écoles résidentielles qui, des années 1870 à 1990, ont séparé de force plus de 150000 enfants autochtones de leur famille dans le but de "tuer l'Indien au sein de l'enfant ». L'objectif assimilationniste reposait en effet sur l'idée colonialiste que les cultures et les croyances spirituelles des Autochtones étaient inférieures (Harper, 2008). En dépit d'un mouvement contemporain de revendication et de résurgence (Alfred et Cornstassel, 2005), de nombreuses communautés des Premières Nations, Métis et Inuits demeurent profondément impactées sur le plan social, culturel et identitaire; et ce à cause des abus et mauvais traitements vécus notamment dans les écoles résidentielles, à cause du génocide culturel subi, du traumatisme intergénérationnel induit, de la privation de droits élémentaires et des multiples discriminations passées et présentes ${ }^{6}$.

Dans ses excuses (Harper, 2008), le ministre insiste sur l'importance de la Commission de Vérité et Réconciliation (CVR, 2015), une commission d'enquête publique qui s'inscrit comme "une démarche de guérison, de réconciliation et de règlement des tristes séquelles laissées par les pensionnats indiens ». À la suite des travaux de cette commission, 94 appels à l'action seront publiés (CVR, 2015) à l'intention de divers acteurs gouvernementaux et de l'Église. Plusieurs ciblent directement la responsabilité du milieu scolaire et éducatif. À travers le Canada, des réformes éducatives ont ainsi vu le jour afin de répondre à cet objectif de réconciliation.

\section{L'émergence de nouveaux standards éducatifs en Alberta}

6 À compter de septembre 2019, les enseignants des écoles élémentaires et secondaires devront désormais pouvoir attester d'une toute nouvelle compétence, relative à «l'application de connaissances de base au sujet des Premières Nations, des Métis et des Inuits » (Alberta Education, 2018). Ils devront notamment mieux faire comprendre à leurs élèves l'histoire et le vécu contemporain des populations autochtones, faire connaître leurs cultures à travers des ressources diversifiées et authentiques. Il est maintenant attendu des enseignants qu'ils puissent nouer des relations efficaces avec des partenaires autochtones, tels que des parents, des aînés ou des gardiens du savoir. Or de telles relations supposent à la fois l'existence d'un réseau, mais aussi la mise en application de protocoles culturels et d'honoraires (Alberta Teachers' Association, 
2019b), ce à quoi les écoles ne sont pas nécessairement habituées. Enfin, le ministère demande désormais à chaque enseignant de s'engager dans sa formation continue pour « améliorer sa compréhension des visions du monde, des croyances culturelles, des langues et des valeurs des Premières Nations, des Métis et des Inuits " (Alberta Éducation, $2018: 4$ ). Par ailleurs, le curriculum scolaire est actuellement en cours de révision pour une plus grande intégration des savoirs et perspectives autochtones dans toutes les matières, de la maternelle à la dernière année du secondaire ${ }^{7}$.

\section{Aperçu de la recherche}

\section{Un projet de recherche axé sur la langue michif}

7 Le michif peut être défini comme la « langue officielle de la nation métisse » (Canadian Geographic, 2018 : 24). Il s'agit également d'une langue en voie de disparition (Moseley, 2010). Historiquement, cette langue est le produit de la rencontre entre les femmes des Premières Nations, essentiellement cries, et les Européens ou Franco-canadiens, principalement francophones, qui vivaient de la traite des fourrures. Les différentes variantes $\mathrm{du}$ michif qui co-existent constituent un continuum de pratiques linguistiques dans lesquelles le français et la langue crie sont plus ou moins prégnants. Le « michif français » (Canadian geographic, 2018:25) ou « français mitchif » (Papen et Bigot, $2010: 201$ ) est ainsi parfois considéré comme un dialecte du français canadien, souvent stigmatisé comme un «mauvais français » (Canadian Geographic, $2018: 25)$. À l'autre extrémité du continuum, se trouve un michif parfois appelé «cri » qui relève essentiellement de cette langue, avec quelques emprunts au français (Iseke, 2013). Au milieu du continuum se trouverait une autre variante, décrite par Rosen (2008), qui emprunte de manière relativement fixe et équilibrée au cri et au français.

8 Dans ce contexte, le postulat de notre recherche était que le michif pourrait constituer un pont entre les Métis et les élèves des écoles francophones et d'immersion française dont la langue d'étude est le français, dans un contexte par ailleurs anglophone majoritaire.

\section{Collaboration avec C. Howse (aînée métisse) et avec R. Beauparlant (enseignant) : des approches à différencier}

9 La recherche, prévue sur plusieurs années, comporte des phases complémentaires impliquant différentes collaborations.

Dans sa phase initiale, elle repose d'abord sur la collecte de récits de vie, soit des entretiens narratifs "centré[s] sur le parcours de vie d'une personne et [...] ses expériences vécues " (Bertaux, 2016:10). Dans un premier temps du projet en effet, une série de souvenirs autobiographiques ont été racontés à la chercheure par $\mathrm{C}$. Howse lors de visites, pendant plus de deux années. En cela, l'approche s'inspire d'une méthodologie de recherche autochtone que Gaudet (2019) nomme la "visiting way methodology ", la collecte des données entrant en synergie avec une éthique de la relation. Comme le souligne Gaudet (2019), cette approche doit être distinguée de la notion de "relationship-building" que l'on retrouve en recherche communautaire et participative : "La notion de "relationship-building ", dans un contexte occidental, est davantage orientée vers la résolution de problèmes pour arriver aux meilleures 
solutions ou résultats possibles, plutôt que de voir l'établissement de relations comme un processus auquel faire confiance en ce qu'il peut aboutir à des résultats inédits et inattendus » (Gaudet, $2019: 59$, n.t.) ${ }^{8}$.

11 Les discussions entre la chercheure et l'aînée ont ainsi abouti à la décision de créer des clips audio, des vidéos ainsi qu'un livre numérique mettant en valeur certains souvenirs et connaissances culturelles chers à C. Howse. Ceux-ci ont été enregistrés/ filmés en langue michif, transcrits, traduits puis édités. Plusieurs personnes de la communauté métisse ont participé à la réalisation de ces ressources, que cela soit pour transcrire et traduire le corpus oral, en proposer une analyse métalinguistique, pour illustrer le livre numérique, ou encore pour apporter une validation quant à la qualité du travail produit ${ }^{9}$. Il est à noter que, dans le contexte de la réconciliation, l'implication de partenaires autochtones est cruciale pour soutenir la légitimité de la recherche et maintenir des relations éthiques entre monde académique et communautés autochtones. Aussi le développement du matériel pédagogique accompagnant les ressources créées avec $\mathrm{C}$. Howse s'est aussi fait en collaboration avec des partenaires Métis. L'approche dite de l'éveil aux langues (Candelier, 2008, 2012), qui a guidé la réalisation du matériel pédagogique, a ainsi été enrichie par l'apport des pédagogies autochtones.

Une fois le matériel pédagogique prêt, la phase de recherche-action-formation (Karsenti et Savoie-Zajc, 2018) a été initiée auprès d'enseignants du primaire. Les buts étaient les suivants :

13 (1) améliorer l'état des connaissances sur l'intégration des perspectives autochtones dans l'enseignement ;

(2) transformer les pratiques éducatives ;

(3) participer au développement professionnel des enseignants ;

(4) améliorer et valider le matériel et la démarche d'enseignement proposés.

Nous avons ainsi travaillé avec quatre écoles et quatre enseignants différents. Toutefois, nous ne retenons ici que notre collaboration avec R. Beauparlant dans la mesure où celui-ci était intéressé à approfondir la discussion sur notre processus de collaboration pour cet article et à contribuer à la diffusion des résultats de la recherche (Lemaire et Beauparlant, 2019, 2020). Soulignons aussi que notre collaboration repose sur une relation antérieure professeur-étudiant et de recherche. En effet, R. Beauparlant, dans le cadre de son Baccalauréat en éducation, a suivi un stage intensif d'enseignement au Togo encadré par E. Lemaire, ce qui nous avait donné l'occasion d'une première collaboration de recherche (Lemaire, Beauparlant, Ani-Meunier et Agbéflé, 2020). R. Beauparlant a par ailleurs eu l'occasion d'occuper des postes d'assistant de recherche alors qu'il suivait sa formation initiale en éducation.

En fonction depuis trois ans dans une école primaire au nord de l'Alberta, R. Beauparlant s'est porté volontaire pour expérimenter la recherche avec sa classe de $6^{\mathrm{e}}$ année (fin du primaire) dans la mesure où il souhaitait se former aux nouvelles exigences ministérielles relatives à l'intégration des réalités autochtones dans l'enseignement. Il a ainsi accepté de se former aux ressources pédagogiques proposées $^{10}$, de planifier les activités de classe avec la chercheure, d'accepter la présence de cette dernière dans la classe, de mettre à disposition les travaux générés par les élèves ainsi que le matériel pédagogique éventuellement ajouté pour répondre aux besoins des élèves. Enfin, R. Beauparlant a accepté de se livrer à un certain nombre 
d'entretiens nrichissant la collecte de données ${ }^{11}$. Trois sessions de travail de une à trois séances de 45 minutes chacune ont eu lieu dans sa classe au cours de l'année académique.

\section{Articulations entre les savoirs de la chercheure et les savoirs de l'enseignant}

19 Avant d'entrer plus en détail dans notre collaboration, rappelons que R. Beauparlant est entré dans le processus de recherche au moment seulement de l'expérimentation en salle de classe, et ce pour permettre de parfaire et valider la démarche et le matériel d'apprentissage co-construits en amont avec divers partenaires Métis. La chercheure joue ainsi un rôle de pont entre communauté métisse et enseignante. Et si les points abordés dans cette partie développent principalement l'articulation des savoirs entre l'enseignant et la chercheure, la communauté autochtone impliquée demeure sousjacente, et nous verrons dans la partie suivante que l'engagement de la communauté est particulièrement importante au moment de diffuser la recherche à plus large échelle.

\section{Structuration de la recherche}

Pour Bourassa et al. (2012: 20), la recherche collaborative (RC) comprend 3 sphères d'intervention, la première étant la structuration, à savoir «l'ensemble des interventions réalisées pour qu'un projet de $\mathrm{RC}$ voit le jour et soit structuré de manière à favoriser l'atteinte des objectifs de la recherche ». Ainsi, en tant que chercheure, nous avons préparé la démarche et les ressources pédagogiques à expérimenter dans un cadre que nous jugions réaliste, basé sur nos connaissances didactiques, notre analyse du curriculum ainsi que nos savoirs quant au milieu éducatif immersif. Un ensemble de négociations sont néanmoins nécessaires afin d'affiner un tel projet. Il s'agit en effet que celui-ci réponde aux besoins réels du terrain. C'est pourquoi nous avons travaillé ensemble, enseignant et chercheure, afin d'élaguer le matériel pédagogique et de choisir les activités qui correspondaient le mieux au niveau des élèves, priorisant également certains objectifs d'apprentissage en fonction de la planification annuelle prévue par l'enseignant. Les connaissances de l'enseignant quant à ses élèves ont permis par ailleurs d'adapter les interventions pédagogiques au rythme d'apprentissage de la classe, de prévoir les adaptations nécessaires pour des pratiques de classe inclusives prenant en compte les besoins spécifiques de certains élèves. Par exemple, pour l'activité perlage ${ }^{12}$, les troubles de l'attention de certains élèves nous ont amenés à décider, en concertation, de préparer fils et aiguilles en avance et d'insister sur une difficulté technique à contourner plutôt que de confronter les élèves à une situation de résolution de problème, comme prévu initialement. Si l'objectif linguistique est perdu de vue (utiliser, en langue seconde, les connecteurs logiques permettant d'exprimer la résolution de problème), les connaissances pragmatiques de l'enseignant quant à sa classe ont permis que l'activité se déroule sans heurt.

21 Par ailleurs, les connaissances de l'enseignant quant à son milieu institutionnel apparaissent essentielles pour le bon déroulement du projet. Une recherche-actionformation est en effet une recherche située, en prise avec des réalités institutionnelles dont le chercheur ne peut avoir de connaissances préalables puisqu'elles sont propres à 
une institution (une école) à laquelle il n'appartient pas. Dans certaines écoles, par exemple, la direction scolaire peut vouloir avoir un droit de regard sur les interventions pédagogiques extérieures, au-delà de l'autorisation préalable donnée. Ainsi, dans notre cas, nous avons dû restreindre le temps accordé au projet et le concentrer sur quelques sessions pour répondre à la crainte du directeur d'école qu'il n'empiète trop sur la progression scolaire des élèves. Comme observé par Bourassa et al. (2012), les cheminements prévus en recherche collaborative sont souvent amenés à être repensés. Aussi, après que le directeur a par la suite assisté à une session d'enseignement et donné une rétroaction très positive sur le projet, nous avons pu constater l'importance de mieux expliciter les dimensions interdisciplinaires et curriculaires du projet pour en augmenter l'attractivité et accroitre les chances que la démarche, à terme, puisse se diffuser à plus large échelle.

\section{Accompagnement et posture collaborative}

22 La sphère de l'accompagnement regroupe, selon Bourassa et al. (2012), l'ensemble des actions et postures déployées par le chercheur au moment de l'expérimentation. Pour le chercheur, il s'agira à la fois d'accepter de partager le contrôle sur la recherche et d'affirmer son positionnement engagé. Dans notre cas, il ne s'agit pas de se limiter à un rôle minimaliste (distribuer des documents par exemple) pour mieux se fondre dans la classe et observer. Co-enseignante, la chercheure accepte que son rôle ne soit pas de produire un savoir neutre et objectif, mais de changer des pratiques et de former, d'effectuer un "travail "avec" et non "sur" » les personnes avec qui on collabore (Bourassa et al., 2012 : 13). Le chercheur fait partie d'une recherche pleinement située, qui se déroule non pas de manière expérimentale, mais "in vivo». Il s'agit de reconnaître que toute mise en œuvre sur le terrain, en salle de classe, avec les élèves, se produit de manière unique, en fonction des participants présents, des dynamiques et interactions spécifiques à un moment donné. La complexité de la réalité scolaire (la nécessité d'avancer dans le programme d'études, les collaborations avec d'autres enseignants ou intervenants, etc.) est telle qu'il n'est pas possible, sur le terrain, de mettre en œuvre la recherche de manière prescriptive et normative. Le chercheur ne saurait par ailleurs exiger de son enseignant partenaire qu'il remette en question la planification de la journée ou de la semaine au seul profit de la recherche. Ainsi, nous n'avons pas pu toujours mettre en œuvre l'intégralité de l'enseignement planifié et, a contrario, nous avons parfois choisi ensemble de « déborder » sur l'enseignement d'une autre matière ${ }^{13}$.

L'enseignant doit quant à lui accepter un lâcher-prise quant à la classe : accepter par exemple de prendre les réponses de tous les élèves ayant levé la main pour permettre de collecter le plus de données possible au lieu de ne donner la parole qu'aux «bons » élèves pour accélérer le rythme. On observera par ailleurs que ce type de pratique permet à l'enseignant de donner une plus grande part à l'évaluation informelle des apprentissages. Il s'agit enfin d'accepter, pour l'enseignant, que le leadership soit partagé, ce qui permettra par ailleurs de modeler auprès des élèves ce qu'est un travail collaboratif. 


\section{Production des connaissances}

24 imputables à la chercheure, quand les savoirs pragmatiques professionnels sont principalement détenus par l'enseignant, sans que cette répartition soit toutefois exclusive. Mais poser une dichotomie stricte serait inexact dans notre cas. On reconnaitra que la chercheure est aussi dotée d'une expérience d'enseignement significative, tout comme on prendra en compte les expériences de jeune chercheur du praticien enseignant.

Ainsi, la collecte des données fut d'autant plus fructueuse que R. Beauparlant était en mesure d'aller au-delà de la simple mise à disposition des travaux réalisés par les élèves lors des sessions en classe. Il a ainsi pris l'initiative de collecter les données qui lui arrivaient en dehors des périodes d'expérimentation. Il a par exemple pris en photo l'artefact qu'une élève lui a présenté fièrement quelque temps après le cours d'initiation au perlage. Il rapportera aussi comment une autre élève, Métisse, a pu établir un dialogue renouvelé avec sa grand-mère autour de cette pratique artistique. R. Beauparlant documentera également une anecdote racontée par une élève qui lui confiera qu'elle s'est rendu compte que sa gardienne était Métisse et qu'elle avait vécu une expérience de scolarisation similaire à celle présentée en classe par C. Howse. L'ajout de ces données est particulièrement intéressant car elles viennent accréditer l'hypothèse que le dispositif permet de créer davantage de liens avec la communauté métisse. Le fait que l'enseignant soit posé comme partenaire de la recherche et non comme sujet aura ainsi permis d'enrichir la collecte de données hors temps d'expérimentation. Un autre exemple serait la proposition, faite par R. Beauparlant, de réaliser un entretien final avec chaque élève pendant le temps de classe, alors qu'en tant que chercheure nous avions choisi de ne pas inclure cette possibilité dans la méthodologie. En effet, créer cet espace de discussion avec les élèves génère d'importantes contraintes pour l'enseignant, mais aussi pour tout chercheur qui doit pouvoir justifier auprès du comité d'éthique de l'université autorisant la recherche que le projet introduit un minimum de perturbation dans la classe. En tant que coenseignants et co-chercheurs, il devient plus aisé de créer cet espace et de le penser collaborativement à la fois comme un temps de collecte de données mais aussi comme une évaluation informelle des apprentissages et un espace d'interaction authentique en langue seconde, dans une perspective enseignante.

En phase d'analyse de données, la co-construction des significations est également de nature à renforcer la validité de la recherche (Bradburry et Reason, 2008 ; Karsenti \& Savoie-Zajc, 2018). Selon Dubet (1994: 233), « tout en répondant à des critères internes de scientificité, la théorie la plus convaincante est celle qui sera la plus proche de l'expérience des acteurs ». Si cette idée est largement reprise dans la littérature (Desgagné et al., 2001; Larouche, 2005 ; Morrissette \& Desgagné, 2009), nous avons choisi d'illustrer ici une situation de convergence des deux points de vue, aussi appelée «situation de double vraisemblance confortée » (Pepin et Desgagné, 2017:131). Du point de vue scientifique et éthique, la démarche de l'éveil aux langues appliquée aux langues des Métis dans une optique de réconciliation ne pouvait, pour la chercheure, que s'enrichir des pédagogies autochtones ${ }^{14}$. Ainsi, le dispositif visait non seulement à familiariser les élèves avec langues et perspectives métisses, mais proposait de prendre en compte des éléments du «comment apprendre » dans une perspective autochtone. 
Parmi les principes pédagogiques convoqués se trouvaient notamment la pédagogie de la terre (Wildcat et al., 2014), l'importance du récit et de la transmission par les aînés, ou encore la valorisation des talents de chaque enfant (Campeau, 2017). En lien avec nos notes de terrain et grâce aux entretiens finaux avec les élèves, nous avons pu mettre en évidence 1) l'intérêt des élèves pour les temps passés hors classe, en forêt, 2) l'empathie des enfants envers l'aînée métisse ayant pris la peine de partager avec eux ses histoires et de répondre à leurs questions, 3) le succès d'un élève à besoins spécifiques excellant dans le perlage quand enseignant et aide-élève avaient postulé à tort que ses troubles de l'attention l'empêcheraient de s'atteler à la tâche. Côté enseignant, les apprentissages parmi les plus marquants, seront justement, pour R. Beauparlant, la prise de conscience (1) que des pratiques autochtones comme la pédagogie de la terre sont bénéfiques aux apprentissages et bien-être de tous les élèves au-delà du projet d'éveil au michif, (2) que l'éducation autochtone s'enrichit de la création de liens avec des personnes de la communauté, et que (3) l'inclusion des élèves à besoins spécifiques peut porter ses fruits à des moments inattendus et qu'il demeure essentiel de planifier ses enseignements en gardant en tête le développement et la valorisation des divers talents des élèves.

Ces exemples, tirés de nos pratiques, illustrent ainsi comment la littérature et méthodologie scientifiques qui soutiennent le dispositif rejoignent les besoins et analyses du praticien.

\section{La posture du chercheur entre enseignants et aînés/ gardiens du savoir autochtones}

Si cet article porte avant tout sur la collaboration entre l'enseignant et la chercheure, il importe de rappeler, encore une fois, que ce dispositif de recherche n'aurait pu être possible sans l'engagement de partenaires autochtones, en particulier de C. Howse. De manière générale, les relations entre chercheurs non autochtones d'une part et aînés ou gardiens du savoir autochtone d'autre part sont marquées par une histoire ethnocentrique et colonialiste qui a vu les savoirs traditionnels autochtones être, dans le champ académique, sous-représentés, mal représentés ou encore méprisés (Battiste, 2011 ; Battiste \& Youngblood, 2000 ; Doxtater, 2004 ; Morgan, 2003). La relation de confiance est à reconstruire, d'autant plus que nombre de savoirs autochtones ont pâti du phénomène d'appropriation culturelle, par lequel les savoirs ont été transmis (souvent de manière erronée et coupée de leur dimension spirituelle), utilisés, commercialisés, publicisés, sans reconnaissance ou profit quelconque pour les individus qui détenaient originalement ces savoirs (Battiste \& Youngblood, 2000 ; Morgan, 2003). Une dimension éthique, liée au contexte de la réconciliation avec les peuples autochtones, caractérise ainsi fortement la démarche de recherche dans son ensemble, depuis la conception du matériel audio, vidéo, textuel et des ressources pédagogiques afférentes, jusqu'à la diffusion de la démarche et du matériel d'enseignement.

Selon Battiste (2007), acquérir un savoir autochtone, même partiellement, n'est nullement anodin. Le processus se fait à travers de longues et multiples conversations avec des aînés, des lieux ${ }^{15}$ et peuples autochtones. Et, de fait, rappelons que ce projet de recherche-action-formation est né autour de thés partagés régulièrement pendant plus de deux ans, dans l'établissement métis ${ }^{16}$ de Kikino. Contrairement aux enseignants 
impliqués dans l'expérimentation en salle de classe, qui ne se sont engagés qu'à une formation minimale via une plateforme d'autoformation en ligne, la chercheure porte une responsabilité directe vis-à-vis de l'aînée et du savoir partagé.

Parmi les dérives possibles, on mentionnera le risque que les ressources pédagogiques, au fil de leur diffusion, soient coupées de leur connexion avec l'épistémologie autochtone, quand la prise en compte de celle-ci fait partie de la responsabilité directe du chercheur. Dès lors, il est essentiel que le dispositif soit partagé sur des réseaux professionnels et autochtones cautionnés par les communautés impliquées et que les ressources créées circulent dans le milieu scolaire accompagnées d'informations pertinentes pour éviter que le matériel soit utilisé de manière superficielle et décontextualisé. Il est également essentiel que les enseignants soient sensibilisés aux pratiques éthiques relatives à l'éducation autochtone afin d'éviter toute situation indue d'appropriation culturelle. Au-delà de l'utilisation des ressources développées dans le cadre de ce projet, il s'agit de susciter une prise de conscience plus large quant à l'intégration des perspectives autochtones. Comme le souligne Battiste (2007: 114) :

«Le défi, pour les éducateurs, demeure donc le suivant : être capable de réfléchir de manière critique sur le système éducatif actuel en se posant la question de savoir quel savoir est représenté à l'école, qui décide de ce qui est offert, quels apprentissages sont valorisés, qui en bénéficie, et de manière plus importante encore, en quoi tout ceci est (ou pas) le résultat d'un processus éthique, approprié (n.t.) ».

31 Ainsi, dans le cadre de notre dispositif, les enseignants expérimentant le matériel pédagogique sont encouragés à créer des connexions avec les communautés autochtones locales, afin de créer ce tissu de relations et d'engagements réciproques autour de la revitalisation des langues et cultures et de la décolonisation des savoirs dans les écoles. C'est ainsi que R. Beauparlant a commencé à développer ses propres relations avec la communauté métisse de Grande Prairie. À terme, au-delà du projet d'éveil au michif expérimenté, on vise l'autonomisation et le renouvellement des pratiques enseignantes, en collaboration avec les communautés, dans une optique de relations durables et de réconciliation.

Enfin, on soulignera simplement, au niveau de la diffusion des connaissances, l'importance, dans notre contexte, « de présenter et diffuser des résultats qui aient une résonnance dans les deux cultures » (Desgagné et al., 2001: 57). Ainsi, s'il est essentiel de communiquer dans la communauté scolaire francophone et francophile pour rejoindre le public des enseignants et les accompagner dans le renouvellement de leurs pratiques, la responsabilité de la chercheure, en lien avec différents organismes et membres de la communauté, sera également de diffuser la recherche de manière à répondre à l'objectif de revitalisation linguistique posé par cette communauté.

\section{Conclusion}

33 En collaboration avec divers partenaires Métis, cette recherche vise à participer à la revitalisation d'une langue autochtone en voie de disparition, le michif. La collaboration avec le milieu scolaire permet quant à elle de faire résonner la voix des Métis auprès des jeunes Canadiens, dans le contexte de la réconciliation. Dans cet article, nous avons voulu mettre en évidence quelques-unes des articulations complexes entre savoirs enseignants et savoirs académiques, mais aussi entre postures et savoirs occidentaux et autochtones, l'accent étant mis sur la complémentarité mais 
aussi sur l'éthique. Alors que le dispositif pédagogique doit être expérimenté dans les prochaines années auprès de davantage d'élèves autochtones ${ }^{17}$, on ne manquera pas de s'intéresser à la singularité de ces nouvelles expériences dont on anticipe qu'elles seront enrichies de perspectives socioculturelles et épistémologiques renouvelées.

\section{BIBLIOGRAPHIE}

Alberta Education (2018). Norme de qualité pour l'enseignement. Récupéré de : https:// education.alberta.ca/media/3576234/nqe-norme-enseignement.pdf.

Alberta Teachers' Association (2019a). Remarques sur la terminologie. Pierres d'assise, v.1. Numéro de catalogue : PD-WT-16bF 2018 04. Repéré à : https://www.teachers.ab.ca/ SiteCollectionDocuments/ATA/For\%20Members/ProfessionalDevelopment/ Walking\%20Together/PD-WT-16gF\%20-\%20Terminology.pdf.

Alberta Teachers' Association (2019b). Protocole relatif aux aînés. Pierres d'assise, v.7. Numéro de catalogue : PD-WT-16gF 2018 04. Récupéré de : https://www.teachers.ab.ca/ SiteCollectionDocuments/ATA/For\%20Members/ProfessionalDevelopment/ Walking\%20Together/PD-WT-16gF\%20-\%20Elder\%20Protocol.pdf.

Alfred, T. et Cornstassel, J. (2005). Being Indigenous : Resurgences against Contemporary Colonialism. Dans Bellamy, R. (dir). The Politics of Identity. Repéré à : http://www.corntassel.net/ being_indigenous.pdf.

Anadón, M. (dir.) (2007). La recherche participative. Multiples regards. Québec : Presses de l'Université du Québec.

Battiste, M. (2011). Reclaiming Indigenous voice and vision. Vancouver : UBC Press.

Battiste, M. (2007). Research ethics for protecting indigenous knowledge and heritage : Institutional and researcher responsibilities. Dans N. K. Denzin \& M. D. Giardina (dir.), Ethical futures in qualitative research : Decolonizing the politics of knowledge. Walnut Creek, CA, US : Left Coast Press, p. 111-132.

Battiste, M. et Youngblood, J. (2000). Protecting Indigenous Knowledge and Heritage : A global challenge. Vancouver : UBC Press.

Bertaux, D. (2016). Le récit de vie. Paris : Armand Colin.

Bourrassa, B., Leclerc, C. et Fournier, G. (2012). Assumer et risquer une posture de recherche collaborative. Dans B. Bourassa et M. Boudjaoui, M. (dir.), Des recherches collaboratives en sciences humaines et sociales (SHS), enjeux, modalités et limites. Québec : Presses de l'université de Laval, p. 13-46.

Bradburry, H. et Reason, P. (2008). Conclusion. Broadening the bandwith of validity : Issues and choice-points for improving the quality of action research. Dans Bradburry H. et Reason, P (dir.), Handbook of action research. Thousand Oaks : Sage Publications, p. 343-351.

Campeau, D. (2017). Pédagogie autochtone. Présentation donnée au Campus Saint-Jean. Edmonton, Université de l'Alberta, 23 mars 2017. 
Canadian Geographic. (2018). Atlas des Peuples Autochtones du Canada. Les Métis. Ottawa : Société géographique royale du Canada.

Candelier, M. (dir.) (2012). CARAP/FREPA. Un Cadre de référence pour les approches plurielles, Compétences et Ressources. Graz : Centre européen pour l'enseignement des langues.

Candelier, M. (2008). Approches plurielles, didactiques du plurilinguisme : le même et l'autre. Les Cahiers de l'Acedle, 5(1), 65-90.

Charlier, B. (2005). Parcours de recherche-action-formation. Revue des sciences de l'éducation, 31(2), 259-272.

Commission de Vérité et de Réconciliation du Canada. (2015). Appels à action. Repéré à : http:// trc.ca/assets/pdf/Calls_to_Action_French.pdf.

Desgagné, S., Bednarz, N., Couture, C., Poirier, L., et Lebuis, P. (2001). L'approche collaborative de recherche en éducation : un rapport nouveau à établir entre recherche et formation. Revue des sciences de l'éducation, 27(1), 33-64.

Doxtater, M. G. (2004). Indigenous Knowledge in the decolonial era. American Indian Quaterly, 28(3-4), 616-633.

Dubet, F. (1994). Sociologie de l'expérience. Paris : Seuil.

Gaudet, C. (2019). Keeoukaywin : The Visiting Way - Fostering an Indigenous Research Methodology. Aboriginal Policy Studies, 7(2), 47-64.

Harper, S. (2008). Présentation d'excuses aux anciens élèves des pensionnats indiens. Repéré à : https:// www.aadnc-aandc.gc.ca/fra/1100100015644/1100100015649.

Herman, M.J. (2011). Working with Elders and Indigenous Knowledge Systems : A reader and guide for places of higher learning. Vernon: J. Charlton Publishing Ltd.

Iseke, J. (2013). Negotiating Métis culture in Michif : Disrupting Indigenous language shift. Decolonization, Indigeneity, Education and Society, 2(2), 92-116.

Karsenti, T. et Savoie-Zajc, L. (2018). La recherche en éducation. Étapes et approches. Montréal : Les Presses de l'Université de Montréal.

Koster, R., Baccar, K. et Lemelin, R.H. (2012). Moving from research ON, to research WITH and FOR Indigenous communities: A critical reflection on community-based participatory research. Le Géographe Canadien, 56(2). Récupéré à : https://onlinelibrary.wiley.com/doi/pdf/10.1111/j.

1541-0064.2012.00428.x.

Larouche, H. (2005). Le double rôle de formatrice et de chercheuse : un point de rencontre pour concilier les besoins de la pratique et de la recherche. Revue des sciences de l'éducation, 31 (2), 335354. doi : doi.org/10.7202/012759ar

Lemaire, E., Beauparlant, R., Ani-Meunier, S. et Agbéflé, K. (2020). Enjeux et réalités de l'enseignement privé au Togo : perspectives d'enseignants (pp. 97-120). Dans J. St Amand (dir.), Amélioration de l'éducation en Afrique sub-saharienne. Québec : Presses de l'université de Laval.

Lemaire, E. et Beauparlant, R. (2020). Comment intégrer les langues et cultures des Métis en classe d'immersion française. Atelier donné lors du Greater Edmonton Teachers' Convention. Edmonton, 28 février 2020.

Lemaire, E. et Beauparlant, R. (2019). Sensibiliser les élèves d'immersion au michif : aperçu d'une recherche-action menée à l'élémentaire. Communication à la Journée de l'ACFAS. Université de l'Alberta, Campus Saint-Jean, 8 mars 2019 
Morgan, D. L. (2003). Appropriation, Appreciation, Accomodation : Indigenous Wisdoms and Knowledges in Higher Education. International Review of Education, 49(1-2), 35-49.

Morissette, J. et Desgagné, S. (2009). Le jeu des positions de savoirs en recherche collaborative : une analyse. Recherches qualitative, 28(2), 118-144.

Moseley, C. (2010). Atlas des langues en danger dans le monde. Paris : Éditions Unesco. Tiré de : www.unesco.org/culture/languages-atlas/fr/atlasmap.html.

National Inquiry into missing and murdered Indigenous Women and Girls (2019). Reclaiming Power and Place. Final Report, vol. 1a. Récupéré à : https://www.mmiwg-ffada.ca/wp-content/uploads/ 2019/06/Final_Report_Vol_1a-1.pdf.

Paillet, P. (1994). Pour une méthodologie de la complexité en éducation : le cas d'une rechercheaction-formation. Revue Canadienne de l'Éducation, 19-3, 215-230.

Papen, R. A. et Bigot, D. (2010). Sontaient, ontvaient, et fontsaient en français michif : variation et systématicité. Dans C. Leblanc (dir.), Vues sur les français d'ici (pp. 201-225). Laval : Presses de l'Université Laval.

Rosen, N. (2008). French-Algonquian Interaction in Canada : A Michif case study. Clinical Linguistics \& Phonetics, 22(8), 610-624.

Pepin, M. et Desgagné, S. (2017). La double vraisemblance au fondement de la collaboration de recherche : retour sur la démarche de coconstruction d'un projet entrepreneurial à l'école primaire. Phronesis, 6(1-2), 126-139.

Strand, K.J., Cutforth, N., Stoecker, R. et Marullo, S. (2003). Community-based research and higher education : Principles and practices. San Francisco : Jossey-Bass.

Wildcat, M., McDonald, M., Irlbacher-Fox, S. et Coulthard, G. (2014). Learning from the land : Indigenous land based pedagogy and decolonization. Decolonization, Indigeneity, Education and Society, 3(3), 1-15.

\section{NOTES}

1. Cet article a été rédigé intégralement par E. Lemaire, avec l'approbation préalable de C. Howse. Il a été discuté puis relu par R. Beauparlant avant soumission.

2. This article has been written by E. Lemaire with previous authorization by C. Howse. The text has been discussed and reviewed by R. Beauparlant before submission.

3. Au Canada, la Constitution reconnaît trois peuples autochtones distincts: les Premières Nations, les Métis et les Inuits.

4. Le féminin est utilisé en référence à l'auteure, le masculin « chercheur » quand il s'agit de la généralisation.

5. Les aînés ou gardiens du savoir autochtones ne sont pas nécessairement des personnes âgées mais des personnes possédant une sagesse et des connaissances culturelles ou traditionnelles qui sont reconnues par les communautés dont ils font partie (Alberta Teachers'Association, 2019b ; Herman, 2011).

6. Il serait impossible de mentionner ici l'ensemble des lois et actes discriminatoires subis de façon systémique par les communautés autochtones au cours de l'histoire et de manière contemporaine. Soulignons toutefois les conclusions du récent rapport sur les femmes et filles autochtones disparues qui conclut au génocide (National Inquiry into missing and murdered Indigenous Women and Girls, 2019). 
7. À noter qu'un changement récent de gouvernement provincial impacte actuellement la réforme des programmes scolaires.

8. En version originale : «In a Western context focuses more on problems and how to arrive at better solutions or outcomes, rather than trusting in a process with unforeseen or unscripted outcomes. »

9. Nous en profitons pour remercier chaleureusement Riplea Lothian, Gabrielle Lamontagne, Athena McKenzie, Maureen Bélanger et Olivia Sammons pour le partage de leurs expertises. Sans leur participation et leurs connaissances, mener un tel projet de recherche n'aurait pas été possible.

10. Une plateforme d'auto-formation accessible en ligne pour les enseignants participant à la recherche a été développée par la chercheure.

11. Le dispositif de recherche prévoit un entretien initial, des entretiens de " débriefing " après chaque séance d'enseignement, ainsi qu'un entretien final.

12. Le perlage est une pratique artistique appartenant à la culture traditionnelle des Métis. C. Howse a choisi de créer une vidéo dans laquelle elle explique comment perler fait partie des ressources expérimentées avec les élèves.

13. À noter que, même s'il existe une marge de liberté pour mener des projets interdisciplinaires ambitieux, l'enseignement en Alberta, au niveau élémentaire, est le plus souvent structuré autour de blocs de 45 minutes dédiés à une discipline particulière.

14. Les élèves ont par exemple fait une analyse métalinguistique de la langue employée par $C$. Howse pour identifier comment français et cri sont tissés ensemble dans ses propos. Ainsi, à partir du tutoriel vidéo sur le perlage, les élèves révisent la nature des mots. La création de liens avec une aînée respectée par vidéo interposée mais aussi par un projet de correspondance, le fait de pratiquer le perlage et de ne pas seulement apprendre à ce sujet, le fait d'aller puiser son inspiration dans la forêt avant de réaliser son artefact, ainsi que le fait d'apprendre sur l'écosystème forestier à cette occasion sont des exemples de comment l'approche pédagogique autochtone a enrichi la démarche d'éveil aux langues, au-delà de la seule analyse linguistique/ métalinguistique du michif.

15. Précisons que, de manière générale, dans l'épistémologie autochtone, les lieux, dont certains ont une forte dimension spirituelle, sont porteurs de sens et portent des savoirs avec lesquels « converser». C'est parce que les lieux sont ainsi chargés en apprentissages que la pédagogie expérientielle et que la pédagogie de la terre sont essentielles dans une approche autochtone de l'enseignement/apprentissage.

16. Les établissements peuvent être pensés, de manière ici simplifiée, comme l'équivalent, pour les Métis, de ce que sont les réserves pour les Premières Nations.

17. Nous prévoyons en effet de proposer, dans les années à venir, cette démarche et ce matériel dans des écoles accueillant un nombre plus élevé d'élèves autochtones, en comparaison avec le contexte des écoles francophones qui, habituellement, dénombrent peu d'élèves s'identifiant comme Premières Nations, Métis ou Inuits. Les écoles que nous contacterons seront situées soit, en ville, dans des quartiers où le taux de population autochtone est élevé, soit en milieu rural, à proximité ou dans les réserves des Premières Nations et établissements métis. Certaines de ces écoles proposent des programmes d'immersion française ou des cours de français de base. 


\section{RÉSUMÉS}

Dans cet article $^{1}$, ancré dans le contexte canadien de la réconciliation avec les peuples autochtones, nous détaillons un projet de recherche-action-formation impliquant à la fois, en amont, une aînée métisse et, en aval, un groupe de 4 enseignants œuvrant à l'élémentaire. Nous nous arrêtons sur les pratiques entre la chercheure et un enseignant spécifique d'une part et sur, d'autre part, la posture de la chercheure qui, à travers le projet de recherche proposé, crée un pont entre les communautés scolaires et les communautés autochtones concernées. Ce texte entend ainsi mettre en évidence quelques-unes des articulations complexes entre savoirs enseignants et savoirs académiques, mais aussi entre postures occidentales et autochtones, l'accent étant mis sur la complémentarité mais aussi sur l'éthique.

In this article ${ }^{2}$, grounded into the Canadian context and into the ongoing reconciliation process between Indigenous and non-Indigenous Peoples, our intent is to discuss a collaborative research involving both a Métis Elder and a group of school teachers. On the one hand, we discuss the complementarities and practicalities implied by the collaboration between the researcher and a specific teacher involved in the research. On the other end, we discuss how the researcher can support building a bridge between local school and Indigenous communities. This text aims to highlight a few complex articulations between teacher and academic knowledge, as well as between Western and Indigenous postures, emphasizing the principle of complementarity and the importance of ethical practices.

\section{INDEX}

Keywords : indigenous, language awareness, teacher training, intercultural, plurilingualism Mots-clés : autochtone, éveil aux langues, formation, interculturel, plurilinguisme

\section{AUTEURS}

\section{EVA LEMAIRE}

University of Alberta

Eva Lemaire est professeure agrégée à la faculté Saint Jean et professeure adjointe à la faculté d'éducation (Department of Educational Policies Study) de l'Université de l'Alberta. Son expertise de recherche est orientée en éducation interculturelle et éducation à la justice sociale. Ses plus récents travaux portent sur l'intégration des savoirs et perspectives autochtones au niveau de la formation des futurs enseignants en contexte francophone minoritaire et au niveau des écoles élémentaires. lemaire[at]ualberta.ca

\section{RENÉ BEAUPARLANT}

École Montrose, Grande-Prairie

René Beauparlant est enseignant à l'école Montrose de Grande Prairie (Alberta). René est originaire du Québec et vit dans l'Ouest Canadien depuis 2012. Très impliqué dans la francophonie albertaine, il est président de l'Association Canadienne Française de l'Alberta au niveau de la région de Grande Prairie ainsi que président du Conseil français, un des 21 conseils de spécialistes de l'Alberta Teachers' Association.

Rene.Beauparlant[at]gppsd.ab.ca 


\section{CÉCILE HOWSE}

Cécile Howse, née Boucher, est Métisse et vit à Kikino, en Alberta. Sa famille, de descendance dénée, crie et francophone, est établie dans la région de Lac La Biche depuis plusieurs décennies. Cécile fut l'épouse de Marshall Howse, avec qui elle a eu dix enfants. Elle est la fille de Vital Boucher et de Madeleine McDonald. Son grand-père paternel se nommait Francis Boucher et sa grand-mère paternelle, Josette Lavallée. Ses grands-parents maternels s'appelaient William et Caroline McDonald. 